\title{
Four-loop QCD MOM beta functions from the three-loop vertices at the symmetric point
}

\author{
Alexander Bednyakov $\circledast^{1,2, \dagger}$ and Andrey Pikelner $\circledast^{1, *}$ \\ ${ }^{1}$ Bogoliubov Laboratory of Theoretical Physics, Joint Institute for Nuclear Research, Joliot-Curie 6, \\ Dubna 141980, Russia \\ ${ }^{2}$ P.N. Lebedev Physical Institute of the Russian Academy of Sciences, \\ Leninskii pr., 5, Moscow 119991, Russia
}

(Received 12 February 2020; accepted 25 March 2020; published 9 April 2020)

\begin{abstract}
For the first time, we compute three-loop contributions to all triple vertices in QCD at the symmetric point. The analytic results are obtained in massless QCD with an arbitrary color group in the Landau gauge. All new loop integrals are expressed in terms of harmonic polylogarithms at the sixth root of unity. These corrections allow us to derive expressions for the four-loop QCD beta function in a set of momentumsubtraction schemes.
\end{abstract}

DOI: 10.1103/PhysRevD.101.071502

\section{INTRODUCTION}

The strong coupling constant $\alpha_{s} \equiv g_{s}^{2} /(4 \pi)$ is a fundamental parameter of QCD. It enters the predictions of many Standard Model observables, e.g., the Higgs production cross section [1] at the LHC, and the uncertainty of $\alpha_{s}$ significantly influences the comparison between theory and experiment. In itself, $\alpha_{s}$ is not a physical observable; its value depends on the scale $Q^{2}$ and the utilized renormalization prescription (or scheme). The freedom in choosing a normalization point manifests itself in "running" governed by the renormalization group $(\mathrm{RG})$ equations

$$
\frac{d \alpha_{s}\left(Q^{2}\right)}{d \ln Q^{2}}=\beta\left(\alpha_{s}\left(Q^{2}\right)\right)
$$

Given the renormalization scheme (RS), one can calculate the $\beta$ function order by order in perturbation theory (PT). To parametrize the strength of strong interactions, one usually uses the $\overline{\mathrm{MS}}$ scheme and quotes the value of $\alpha_{s}^{(5)}\left(M_{Z}\right)=0.1179(10)$ [2] defined in effective QCD with $n_{f}=5$ massless flavors and evaluated at the $Z$-boson mass. This definition is of great convenience when studying inclusive observables dominated by short-distance effects. For almost 20 years the four-loop $\beta^{\overline{\mathrm{MS}}}[3,4]$ in the $\overline{\mathrm{MS}}$ scheme has been utilized in state-of-the-art QCD

\footnotetext{
*Corresponding author.

pikelner@theor.jinr.ru

bednya@theor.jinr.ru
}

Published by the American Physical Society under the terms of the Creative Commons Attribution 4.0 International license. Further distribution of this work must maintain attribution to the author(s) and the published article's title, journal citation, and DOI. Funded by SCOAP. calculations, and only recently the five-loop result [5-8] became available in the literature. The latter allows one to significantly improve the agreement between $\alpha_{s}$ determinations (see, e.g., Ref. [2] for details) in precision measurements carried out at different energy scales.

At lower energies other $\alpha_{s}$ definitions can be more convenient. For example, in lattice QCD one can introduce the strong coupling as a particular RG-invariant combination (the so-called invariant charge) of the vertex and twopoint Green functions evaluated at fixed external momenta in a fixed (usually Landau) gauge (see, e.g., Refs. $[9,10]$ ). Since lattice results [11] give rise to one of the most precise determinations of $\alpha_{s}^{(5)}\left(M_{Z}\right)$, it is important to study the strong coupling and its running in the momentum-subtraction (MOM) schemes. In these RSs the vertex functions and propagators are normalized in such a way that at a certain kinematic point there are no corrections beyond the tree level. As a consequence, the renormalized couplings coincide with the corresponding invariant charges and can be directly compared to the nonperturbative lattice results.

Contrary to the $\overline{\mathrm{MS}}$ scheme, in the MOM scheme one needs to know the Green functions beyond the divergent terms. Some choices of kinematics can make the calculation quite challenging, especially of vertices. In this paper, we consider three-point functions that depend on momenta $p_{1}, p_{2}$, and $q=p_{1}+p_{2}$ (see Fig. 1) and utilize the symmetric normalization point (SMOM) in the Euclidean region $p_{1}^{2}=p_{2}^{2}=q^{2}=-Q^{2}$. One-loop QCD renormalization in the SMOM scheme in a general linear gauge has been known for quite a long time, following the pioneering work in Ref. [12]. At the moment, only two-loop expressions for the QCD vertices in this kinematics are available in the literature in numerical [13] and analytical [14] forms, allowing one to find three-loop $[13,15]$ strong-coupling beta functions in the SMOM RS. In our work, we exploit modern Feynman-integral 


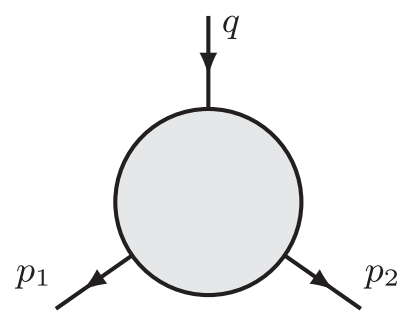

FIG. 1. For symmetric point integrals $p_{1}^{2}=p_{2}^{2}=q^{2}$, and for auxiliary integrals we use $p_{1}^{2}=p_{2}^{2}, q^{2}=x p_{1}^{2}$.

evaluation methods to improve these results by one more order of PT. To simplify our calculation, we routinely use the Landau gauge, in which the gluon propagator is transversal and there is no need to consider the gaugeparameter renormalization.

In the context of perturbative QCD (pQCD) one can relate renormalized couplings $a_{\text {ren }} \equiv \alpha_{s}^{\text {ren }} /(4 \pi)$ defined in different RSs. In what follows, we consider $a_{\text {ren }}=\left\{a_{\text {ggg }}\right.$, $\left.a_{\mathrm{ccg}}, a_{\mathrm{qqg}}\right\}$, which satisfy

$$
\begin{aligned}
\mu^{-2 \varepsilon} a_{\text {bare }} & =Z_{a_{\mathrm{ren}}} a_{\text {ren }}=\left[\frac{Z_{\mathrm{ggg}}^{2}}{Z_{\mathrm{gg}}^{3}}\right] a_{\mathrm{ggg}} \\
& =\left[\frac{Z_{\mathrm{ccg}}^{2}}{Z_{\mathrm{cc}}^{2} Z_{\mathrm{gg}}}\right] a_{\mathrm{ccg}}=\left[\frac{Z_{\mathrm{qqg}}^{2}}{Z_{\mathrm{qq}}^{2} Z_{\mathrm{gg}}}\right] a_{\mathrm{qqg}},
\end{aligned}
$$

with $a_{\text {bare }}$ being the bare coupling defined in the dimensionally regularized theory with $d=4-2 \varepsilon$. The bare coupling is divergent and is related to the renormalized ones $a_{\text {ren }}$ via divergent factors $Z_{a_{\text {ren }}}$. The latter are combinations of the renormalization constants of the three-gluon $\left(Z_{\text {ggg }}\right)$, ghost-gluon $\left(Z_{\mathrm{ccg}}\right)$, and quark-gluon $\left(Z_{\mathrm{qqg}}\right)$ vertices together with those of the gluon $\left(Z_{\mathrm{gg}}\right)$, ghost $\left(Z_{\mathrm{cc}}\right)$, and quark $\left(Z_{\mathrm{qq}}\right)$ fields.

The vertex renormalization constants denoted collectively by $Z_{V}$ are obtained order by order in perturbation theory from the bare $\Gamma_{\text {bare }}^{V}$ vertices by making sure that the renormalized counterpart $\Gamma_{\text {ren }}^{V}$,

$$
\begin{aligned}
\Gamma_{\text {ren }}^{V}\left(q_{i}, a_{\text {ren }}\right) & =Z_{V}\left(a_{\text {ren }}\right) \Gamma_{\text {bare }}^{V}\left(q_{i}, a_{\text {bare }}\right), \\
\mu^{-2 \varepsilon} a_{\text {bare }} & =Z_{a_{\text {ren }}} a_{\text {ren }},
\end{aligned}
$$

is finite for all external momenta $q_{i}$ and satisfies certain normalization conditions.

In the $\overline{\mathrm{MS}}$ scheme only divergent terms are subtracted, and one can prove that $a_{\overline{\mathrm{MS}}}=a_{\mathrm{ggg}}^{\overline{\mathrm{MS}}}=a_{\mathrm{ccg}}^{\overline{\mathrm{MS}}}=a_{\mathrm{qqg}}^{\overline{\mathrm{MS}}}$. On the contrary, in the SMOM schemes one also subtracts finite terms and requires that there are no $\mathcal{O}\left(a_{\text {ren }}\right)$ corrections to a particular $\Gamma_{\text {ren }}^{V}$ at the symmetric point, characterized by momentum $Q^{2}$. To avoid the appearance of $\log \left(Q^{2} / \mu^{2}\right)$ in the renormalization constants, it is convenient to choose $Q^{2}=\mu^{2}$.
The vertex functions of our interest have color and spacetime indices and one can decompose them in terms of basis tensors with Lorentz-invariant coefficients (form factors). The choice of the basis is not unique and we make use of a decomposition that is valid for the symmetric point [14]. To save space, we present here only terms [13] that are relevant for the definition of the required vertex renormalization constants (3):

$$
\begin{gathered}
\Gamma_{\mu}^{a b c}\left(p_{1}, p_{2}\right)=-i g_{s} f^{a b c}\left(p_{1}^{\nu} g_{\nu \mu} \Gamma^{\mathrm{ccg}}\left(-\mu^{2}\right)+\ldots\right), \\
\Gamma_{\mu \nu \rho}^{a b c}\left(p_{1}, p_{2}\right)=i g_{s} f^{a b c}\left(T_{\mu \nu \rho} \Gamma^{\mathrm{ggg}}\left(-\mu^{2}\right)+\ldots\right), \\
\Gamma_{\mu, i j}^{a}\left(p_{1}, p_{2}\right)=g_{s} T_{i j}^{a}\left(\gamma_{\mu} \Gamma^{\mathrm{qqg}}\left(-\mu^{2}\right)+\ldots\right) .
\end{gathered}
$$

Here all momenta are assumed to be outgoing, $p_{1}$ in Eq. (4) corresponds to the antighost, and $T_{\mu \nu \rho}=$ $g_{\mu \nu}\left(p_{1}-p_{2}\right)_{\rho}+\cdots$ represents the tensor that enters into the tree-level three-gluon vertex. The $\mathrm{SU}(\mathrm{N})$ generators $T_{i j}^{a}$ in the quark-gluon vertex (6) satisfy $\left[T^{a}, T^{b}\right]_{i j}=i f^{a b c} T_{i j}^{c}$ with structure constants $f^{a b c}$.

The expressions for the bare form factors $\Gamma_{\text {bare }}^{V}$ with $V=$ $\{$ ggg, ccg, qqg\} are extracted from the tensor vertices by means of the projectors given in Ref. [14]. To define $a_{\text {ggg }}$ in the respective SMOM scheme (MOMggg) via Eq. (2), we require that at the symmetric point $\Gamma_{\mathrm{ren}}^{\mathrm{ggg}}\left(-\mu^{2}\right)=1$, i.e., $Z_{\mathrm{ggg}}{ }^{-1}=\Gamma_{\mathrm{bare}}^{\mathrm{ggg}}\left(-\mu^{2}\right)$. In the same way one can relate the bare coupling $a_{\text {bare }}$ to $a_{\mathrm{ccg}}$ or $a_{\mathrm{qqg}}$ by requiring that either $\Gamma_{\text {ren }}^{\mathrm{ccg}}\left(-\mu^{2}\right)=1$ (MOMh) or $\Gamma_{\text {ren }}^{\mathrm{qqg}}\left(-\mu^{2}\right)=1$ (MOMq). Since field renormalization constants also enter into Eq. (2), we have to impose conditions on $Z_{\mathrm{gg}}, Z_{\mathrm{cc}}$, and $Z_{\mathrm{qq}}$ in the SMOM scheme: there should be no corrections to the corresponding tree-level propagator for the external momentum $q^{2}=-\mu^{2}$.

From Eq. (2) one can deduce that two renormalization prescriptions for $a_{\mathrm{ren}}$, say $a_{R}$ and $a_{\overline{\mathrm{MS}}}$, are related via finite correction factors $X_{R}$ :

$a_{R}=\left(Z_{a_{R}} / Z_{a_{\overline{\mathrm{MS}}}}\right) a_{\overline{\mathrm{MS}}} \equiv a_{\overline{\mathrm{MS}}} X_{R}=a_{\overline{\mathrm{MS}}}\left[1+\sum_{l} X_{R}^{(l)} a_{\overline{\mathrm{MS}}}^{l}\right]$.

Given $X_{R}$ at $L$ loops, one can determine the $(L+1)$ MOMscheme beta functions from $\beta \overline{\mathrm{MS}}$ via a relation that is valid in the Landau gauge,

$\beta_{R} \equiv \frac{d a_{R}}{d \ln \mu^{2}}=\frac{\partial a_{R}\left(a_{\overline{\mathrm{MS}}}\right)}{\partial a_{\overline{\mathrm{MS}}}} \cdot \beta_{\overline{\mathrm{MS}}}\left(a_{\overline{\mathrm{MS}}}\right), \quad a_{\overline{\mathrm{MS}}}=a_{\overline{\mathrm{MS}}}\left(a_{R}\right)$,

where in the final step we invert Eq. (7) to express $\beta_{R}$ in terms of $a_{R}$. The main aim of this paper is to calculate the three-loop corrections $X_{R}^{(3)}$ to the relations (7) between 
$a_{\mathrm{ggg}}, a_{\mathrm{ccg}}, a_{\mathrm{qqg}}$, and $a_{\overline{\mathrm{MS}}}$. As one of the applications of our result, we use the four-loop beta function in the $\overline{\mathrm{MS}}$ scheme $[3,4]$ to find the corresponding beta functions $\beta_{\mathrm{ggg}}, \beta_{\mathrm{ccg}}$, and $\beta_{\text {qqg }}$ in the considered SMOM schemes.

\section{DETAILS OF THE CALCULATION}

We generate Feynman diagrams with DIANA [16], and obtain 8, 106, and 2382 graphs for the $\Gamma_{\text {ren }}^{\text {ggg }}$ three-gluon vertex at one, two, and three loops, respectively. Both ghost-gluon and quark-gluon vertices give rise to 2,33 , and 688 diagrams at the same loop levels. After the application of projectors [14] and taking fermion and color traces [17], we are left with scalar Feynman integrals, which we reduce to a set of two one-loop, eight two-loop, and 51 three-loop master integrals by means of REDUZE2 [18] and FIRE6 [19].

The main challenge is calculating the full set of threeloop three-point integrals in SMOM kinematics, which is not available in the literature. To compute master integrals, we rely on the linear reducibility of massless vertex-type integrals with arbitrary off-shell momenta, which is proven to take place up to three-loop order [20,21]. Due to the latter property, for our integrals in more restricted kinematics we choose two strategies of evaluation. First of all, we derive a new basis of finite master integrals [22] and try to compute them directly by means of the Hyperint package [23] in terms of generalized polylogarithms (GPLs). Unfortunately, we are unable to calculate the most complicated integrals and instead use another strategy based on the solution of the system of differential equations (DEs). The method of DEs cannot be applied directly to the calculation of single scale integrals we are interested in, and we construct a set of auxiliary integrals with arbitrary external $q^{2}=x p_{1}^{2}$ (Fig. 1). After switching to a new variable $z, x=2-z-1 / z$, we reduce the original $\mathrm{DE}$ system to the so called $\varepsilon$-form [24]. Due to the presence of singularities at complex points, we make use of the EPSILON package [25], which is capable of dealing with the latter. The obtained system in the $\varepsilon$-form is easily solved order by order in the $\varepsilon$ expansion. The solution is given by linear combinations of GPLs $G\left(a_{1}, \ldots, a_{n} ; z\right)$, where $a_{i}$ correspond to different sixth roots of unity, with a number of unknown constants to be fixed from boundary conditions. The latter can be obtained by matching the expansion in the limit $q^{2} \rightarrow 0$, corresponding to $z \rightarrow 1$, with the explicit result of large-momentum asymptotic expansion. Since $z=1$ is a singular point of the DEs, naive Taylor expansion is not sufficient to fix all of the constants and we exploit the EXP package $[26,27]$ to generate the series in terms of massless propagators. We compute the latter by the MINCER package $[28,29]$ keeping exact dependence ${ }^{1}$ on the space-time dimension variable.

In this way, we obtain the analytic results for auxiliary integrals depending on $z$. Taking the limit $z \rightarrow e^{i \pi / 3}$, which is regular and corresponds to SMOM kinematics, we compute the required single-scale master integrals. The correctness of the analytic calculation is verified numerically using pysecDec [30]. Substituting the integrals in the expressions for the three-loop $\Gamma_{\text {bare }}^{V}$ and expanding in $\varepsilon$ up to the necessary order, we see that the maximal transcendental weight in the final result is, as expected, $2 L=6$, with $L$ being the loop order. Using the basis and the reduction rules from Ref. [31], we are able to simplify the expressions for $\Gamma_{\text {bare }}^{V}$ significantly. As a consequence, we obtain the SMOM renormalization constants, conversion factors (7), and the beta functions (8) in a rather compact form.

\section{RESULTS AND CONCLUSION}

All of the necessary renormalization constants up to three-loop order were calculated iteratively via Eq. (3). We also reproduced the well-known three-loop $\overline{\mathrm{MS}}$ expressions, required to derive the relations (7) between the SMOM couplings and $a_{\overline{\mathrm{MS}}}$ evaluated at the same scale $Q^{2}=\mu^{2}$. To save space, we present the results in numerical form with all QCD color factors substituted explicitly:

$$
\begin{aligned}
X_{\mathrm{ccg}}= & 1+a_{\overline{\mathrm{MS}}}\left(18.54827536-1.111111111 n_{f}\right)+a_{\overline{\mathrm{MS}}}^{2}\left(641.9400677-85.55595017 n_{f}+1.234567901 n_{f}^{2}\right) \\
& +a_{\overline{\mathrm{MS}}}^{3}\left(26810.13185-5350.674817 n_{f}+240.8472277 n_{f}^{2}-1.371742112 n_{f}^{3}\right), \\
X_{\mathrm{qqg}}= & +a_{\overline{\mathrm{MS}}}\left(16.71577458-1.11111111 n_{f}\right)+a_{\overline{\mathrm{MS}}}^{2}\left(472.1590958-83.11121681 n_{f}+1.234567901 n_{f}^{2}\right) \\
+ & a_{\overline{\mathrm{MS}}}^{3}\left(16997.21982-4340.986026 n_{f}+228.6939963 n_{f}^{2}-1.371742112 n_{f}^{3}\right), \\
X_{\mathrm{ggg}}= & 1+a_{\overline{\mathrm{MS}}}\left(26.49248887-3.416806434 n_{f}\right)+a_{\overline{\mathrm{MS}}}^{2}\left(960.4627178-202.0850109 n_{f}+7.687393017 n_{f}^{2}\right) \\
& +a_{\overline{\mathrm{MS}}}^{3}\left(42285.00716-12133.42891 n_{f}+902.7134506 n_{f}^{2}-14.34154686 n_{f}^{3}\right) .
\end{aligned}
$$

\footnotetext{
${ }^{1}$ Available at https://www.nikhef.nl/ form/maindir/packages/mincer/mincerex.tgz.
} 
The analytic results for the general gauge group are available as Supplemental Material [32] and have several remarkable properties. We managed to simplify the twoloop part [15] and expressed it solely in terms of powers of $\pi$, odd $\zeta$ values, and two polygamma functions $\psi^{(1)}(1 / 3)$ and $\psi^{(3)}(1 / 3)$. It turns out that to write down the three-loop contribution we only need to introduce three additional constants: $\psi^{(5)}(1 / 3)$, and two combinations $H_{5}$ and $H_{6}$ of GPLs with uniform transcendental weights five and six, respectively. Evaluating $H_{5,6}$ numerically [33] with high precision and using the PSLQ algorithm [34], we reconstructed the new constants ${ }^{2}$ through a more restricted basis [36] of real parts of harmonic polylogarithms of the argument $e^{i \pi / 3}$.

As a first application of Eqs. (9), (10), and (11) we obtain a set of SMOM beta functions via Eq. (8), thus extending the results of Ref. [15] to four-loop order:

$$
\begin{aligned}
\beta_{\mathrm{ccg}}= & \beta_{\mathrm{uni}}\left(a_{\mathrm{ccg}}\right)-a_{\mathrm{ccg}}^{4}\left(2813.492952-617.6471546 n_{f}+21.50281811 n_{f}^{2}\right) \\
& -a_{\mathrm{ccg}}^{5}\left(96089.34786-23459.32128 n_{f}+1735.992218 n_{f}^{2}-33.24145137 n_{f}^{3}\right), \\
\beta_{\mathrm{qqg}}= & \beta_{\mathrm{uni}}\left(a_{\mathrm{qqg}}\right)-a_{\mathrm{qqg}}^{4}\left(1843.652731-588.6548459 n_{f}+22.58781183 n_{f}^{2}\right) \\
& -a_{\mathrm{qqg}}^{5}\left(68529.68547-15466.43194 n_{f}+1093.568841 n_{f}^{2}-18.85323795 n_{f}^{3}\right), \\
\beta_{\mathrm{ggg}}= & \beta_{\mathrm{uni}}\left(a_{\mathrm{ggg}}\right)-a_{\mathrm{ggg}}^{4}\left(1570.9844+0.56592607 n_{f}-67.089536 n_{f}^{2}+2.6581155 n_{f}^{3}\right) \\
- & a_{\mathrm{ggg}}^{5}\left(94167.261-27452.645 n_{f}+4152.5388 n_{f}^{2}-543.68484 n_{f}^{3}+20.429348 n_{f}^{4}\right),
\end{aligned}
$$

with $\beta_{\text {uni }}(a)=-a^{2}\left(11-2 / 3 n_{f}\right)-a^{3}\left(102-38 / 3 n_{f}\right)$ being the universal two-loop scheme-independent contribution.

The obtained formulas can be used in a number of ways. For example, one can improve the precision of matching between the lattice and $\mathrm{pQCD}$ results. In addition, the possibility to switch from one RS to another in truncated PT series for observables, or more generally RG-invariant quantities (see, e.g., Refs. [37,38]), provides us with an additional handle on theoretical uncertainties beyond

\footnotetext{
${ }^{2} \mathrm{~A}$ similar set of constants generated by cyclotomic polylogarithms was considered in Ref. [35].
}

simple scale variation. Moreover, the computed integrals can also be used in SMOM calculations of more complicated three-point Green functions with operator insertions, e.g., for studies of the light-quark masses as in Refs. $[39,40]$.

\section{ACKNOWLEDGMENTS}

We thank A. Kotikov, V. Magerya, and S. Mikhailov for fruitfull discussions. The work of A. P. is supported by the Foundation for the Advancement of Theoretical Physics and Mathematics "BASIS." The work of A. B. is supported by the Grant of the Russian Federation Government, Agreement No. 14.W03.31.0026 from 15.02.2018.
[1] C. Anastasiou, C. Duhr, F. Dulat, F. Herzog, and B. Mistlberger, Higgs Boson Gluon-Fusion Production in QCD at Three Loops, Phys. Rev. Lett. 114, 212001 (2015).

[2] M. Tanabashi et al. (Particle Data Group), Review of particle physics, Phys. Rev. D 98, 030001 (2018).

[3] T. van Ritbergen, J. A. M. Vermaseren, and S. A. Larin, The Four loop beta function in quantum chromodynamics, Phys. Lett. B 400, 379 (1997).

[4] M. Czakon, The Four-loop QCD beta-function and anomalous dimensions, Nucl. Phys. B710, 485 (2005).

[5] P. A. Baikov, K. G. Chetyrkin, and J. H. Kühn, Five-Loop Running of the QCD Coupling Constant, Phys. Rev. Lett. 118, 082002 (2017).
[6] T. Luthe, A. Maier, P. Marquard, and Y. Schroder, The fiveloop Beta function for a general gauge group and anomalous dimensions beyond Feynman gauge, J. High Energy Phys. 10 (2017) 166.

[7] F. Herzog, B. Ruijl, T. Ueda, J. A. M. Vermaseren, and A. Vogt, The five-loop beta function of Yang-Mills theory with fermions, J. High Energy Phys. 02 (2017) 090.

[8] K. G. Chetyrkin, G. Falcioni, F. Herzog, and J. A. M. Vermaseren, Five-loop renormalisation of QCD in covariant gauges, J. High Energy Phys. 10 (2017) 179; Addendum, 12 (2017) 006.

[9] Ph. Boucaud, M. Brinet, F. De Soto, V. Morenas, O. Pène, K. Petrov, and J. Rodríguez-Quintero, Three-gluon running coupling from lattice QCD at $N_{f}=2+1+1$ : a 
consistency check of the OPE approach, J. High Energy Phys. 04 (2014) 086.

[10] S. Zafeiropoulos, P. Boucaud, F. De Soto, J. RodríguezQuintero, and J. Segovia, Strong Running Coupling from the Gauge Sector of Domain Wall Lattice QCD with Physical Quark Masses, Phys. Rev. Lett. 122, 162002 (2019).

[11] S. Aoki et al. (Flavour Lattice Averaging Group), FLAG review 2019, Eur. Phys. J. C 80, 113 (2020).

[12] W. Celmaster and R. J. Gonsalves, The renormalization prescription dependence of the QCD coupling constant, Phys. Rev. D 20, 1420 (1979).

[13] K. G. Chetyrkin and T. Seidensticker, Two loop QCD vertices and three loop MOM beta functions, Phys. Lett. B 495, 74 (2000).

[14] J. A. Gracey, Two loop QCD vertices at the symmetric point, Phys. Rev. D 84, 085011 (2011).

[15] J. A. Gracey, Three loop QCD MOM beta-functions, Phys. Lett. B 700, 79 (2011).

[16] M. Tentyukov and J. Fleischer, A Feynman diagram analyzer DIANA, Comput. Phys. Commun. 132, 124 (2000).

[17] T. van Ritbergen, A. N. Schellekens, and J.A. M. Vermaseren, Group theory factors for Feynman diagrams, Int. J. Mod. Phys. A 14, 41 (1999).

[18] A. von Manteuffel and C. Studerus, Reduze 2-distributed feynman integral reduction, arXiv:1201.4330.

[19] A. V. Smirnov and F. S. Chuharev, FIRE6: Feynman integral reduction with modular arithmetic, Comput. Phys. Commun. 247, 106877 (2020).

[20] F. Chavez and C. Duhr, Three-mass triangle integrals and single-valued polylogarithms, J. High Energy Phys. 11 (2012) 114.

[21] E. Panzer, On hyperlogarithms and Feynman integrals with divergences and many scales, J. High Energy Phys. 03 (2014) 071.

[22] A. von Manteuffel, E. Panzer, and R. M. Schabinger, A quasi-finite basis for multi-loop feynman integrals, J. High Energy Phys. 02 (2015) 120.

[23] E. Panzer, Algorithms for the symbolic integration of hyperlogarithms with applications to Feynman integrals, Comput. Phys. Commun. 188, 148 (2015).

[24] J. M. Henn, Multiloop Integrals in Dimensional Regularization Made Simple, Phys. Rev. Lett. 110, 251601 (2013).

[25] M. Prausa, epsilon: A tool to find a canonical basis of master integrals, Comput. Phys. Commun. 219, 361 (2017).

[26] R. Harlander, T. Seidensticker, and M. Steinhauser, Complete corrections of Order alpha alpha-s to the decay of the $\mathrm{Z}$ boson into bottom quarks, Phys. Lett. B 426, 125 (1998).
[27] T. Seidensticker, Automatic application of successive asymptotic expansions of Feynman diagrams, in 6th International Workshop on New Computing Techniques in Physics Research: Software Engineering, Artificial Intelligence Neural Nets, Genetic Algorithms, Symbolic Algebra, Automatic Calculation (AIHENP 99) Heraklion, Crete, Greece, 1999 (1999), arXiv:hep-ph/9905298.

[28] S. G. Gorishnii, S. A. Larin, L. R. Surguladze, and F. V. Tkachov, Mincer: Program for multiloop calculations in quantum field theory for the schoonschip system, Comput. Phys. Commun. 55, 381 (1989).

[29] S. A. Larin, F. V. Tkachov, and J. A. M. Vermaseren, The FORM version of MINCER, Report No. NIKHEF-H-91-18, 1991.

[30] S. Borowka, G. Heinrich, S. Jahn, S. P. Jones, M. Kerner, J. Schlenk, and T. Zirke, pySecDec: a toolbox for the numerical evaluation of multi-scale integrals, Comput. Phys. Commun. 222, 313 (2018).

[31] J. M. Henn, A. V. Smirnov, and V. A. Smirnov, Evaluating multiple polylogarithm values at sixth roots of unity up to weight six, Nucl. Phys. B919, 315 (2017).

[32] See Supplemental Material at http://link.aps.org/ supplemental/10.1103/PhysRevD.101.071502 for computer-readable analytic results for beta functions and conversion factors.

[33] J. Vollinga and S. Weinzierl, Numerical evaluation of multiple polylogarithms, Comput. Phys. Commun. 167, 177 (2005).

[34] H. Ferguson, D. Bailey, and S. Arno, Analysis of pslq, an integer relation finding algorithm, Math. Comput. 68, 351 (1999).

[35] J. Ablinger, J. Blumlein, and C. Schneider, Harmonic sums and polylogarithms generated by cyclotomic polynomials, J. Math. Phys. (N.Y.) 52, 102301 (2011).

[36] B. A. Kniehl, A. F. Pikelner, and O. L. Veretin, Three-loop massive tadpoles and polylogarithms through weight six, J. High Energy Phys. 08 (2017) 024.

[37] J. A. Gracey, Momentum subtraction and the $R$ ratio, Phys. Rev. D 90, 094026 (2014).

[38] F. Herzog, B. Ruijl, T. Ueda, J. A. M. Vermaseren, and A. Vogt, On Higgs decays to hadrons and the R-ratio at $\mathrm{N}^{4} \mathrm{LO}$, J. High Energy Phys. 08 (2017) 113.

[39] L. G. Almeida and C. Sturm, Two-loop matching factors for light quark masses and three-loop mass anomalous dimensions in the RI/SMOM schemes, Phys. Rev. D 82, 054017 (2010).

[40] J. A. Gracey, RI'/SMOM scheme amplitudes for quark currents at two loops, Eur. Phys. J. C 71, 1567 (2011). 